\title{
The future of flavonoid research
}

\author{
Colin D. Kay* \\ Diet and Health Group, School of Medicine, MED 1, Floor 2, University of East Anglia, Norwich NR4 7TJ, UK
}

(Received 27 January 2010 - Revised 10 May 2010 - Accepted 14 June 2010)

There are insufficient data to provide conclusive evidence on the health effects of most flavonoid subclasses. Future research of polyphenol bioactivity requires a more complete understanding of their intake, bioavailability and metabolism. The following summarises the limitations of polyphenol research as described across various reviews throughout the literature and presents the key requirements for future research. These include establishing the effects of processing, bioavailability and metabolism, developing physiologically appropriate in vitro models, standard methods of analysis and appropriate clinical biomarkers. The future of flavonoid research will undoubtedly depend upon the resolve of these issues, and although the field has shown continuous progress for many years, progress will likely slow if these challenges are not met

Flavonoid: Bioactivity: Bioavailability: Metabolism

An ever-expanding amount of scientific evidence supports a protective effect of polyphenols on chronic degenerative diseases $^{(1-5)}$. Over the past decade, a significant amount of short duration human intervention studies have been conducted with the goal of establishing the exact bioefficacy of various polyphenol subclasses. A recent meta-analysis of these randomised controlled trials provided a 'snapshot' of the available literature in relation to the relative effectiveness of the different flavonoid subclasses in protecting against $\mathrm{CVD}^{(3)}$. The review detailed a significant amount of evidence to support the activity of cocoa catechins on systolic blood pressure and flow-mediated dilatation; however, there was insufficient consistent evidence to provide conclusions regarding most other subclasses of flavonoid. Additionally, although there were sufficient data available to draw conclusions for a few biomarkers of disease, such as flow-mediated dilatation, blood pressure and LDLcholesterol, there were insufficient studies to draw definitive conclusions for most other cardiovascular end points ${ }^{(3)}$. The apparent shortcomings in the intervention literature primarily stem from a lack of standardised biomarkers of intake, a lack of studies using pure compounds, a lack of long duration clinical interventions and a lack of studies quantifying flavonoid dose, blood level or pharmacokinetics.

Establishing the biological activities of phytochemicals (including flavonoids/polyphenols) is dependent on the complete understanding of their intake, absorption, metabolism and excretion; however, to date, this has only been realised for a limited few structures. As suggested and observed by Scalbert $\&$ Williamson $^{(6)}$, the (in vitro) plasma radical scavenging capacity following flavonoid interventions suggests that a significant amount of unknown metabolites must be present in the blood $^{(6)}$, implicating a much greater absorption and bioavailability than perceived. These unknown metabolites are still a matter of much debate for many of the flavonoid subclasses and most likely contribute to the bioactivity of flavonoids. Similarly, a review by Manach et al. ${ }^{(7)}$ concluded that establishing the differences in flavonoid metabolism was essential for the future design and interpretation of intervention studies investigating the health effects of flavonoids.

The level of consumption of dietary flavonoids is still a matter of much debate, with the total intake of dietary phenols and flavonoids generally approximated to be about $1 \mathrm{~g} / \mathrm{d}$, and phenolic acids and complex polymers are estimated to account for a significant proportion of this estimated amount ${ }^{(6)}$. This estimation is essentially unchanged since it was presented by Kuhnau et $a{ }^{\left({ }^{(8)}\right.}$. As conceded in a recent review by Spencer et al. ${ }^{(9)}$, it has proven extremely difficult to quantitatively establish the benefits afforded by flavonoids as a result of their great diversity in foods, the limited data available regarding their content in foods and a limited understanding of their metabolic fates ${ }^{(9)}$.

Currently, the United states Department of Agriculture maintains a national database for food composition which includes values for many polyphenols, including flavonoids, proanthocyanidins and isoflavones ${ }^{(10)}$. However, this database is still a long way from being complete, which is understandable when you factor in the complexity of fruit and vegetable phytochemical content across species, cultivars and growing and processing conditions. There are other food composition databases arising which contain comprehensive analysis of the flavonoid content of many foods, including a database managed by the Institut National de la Recherche Agronomique (Phenol-Explore; www.phenol-explorer.eu) ${ }^{(5)}$ and a database within the European Food Information Resources Network ${ }^{(11)}$. In the future, the use of these databases in combination could provide a very powerful tool for the design and interpretation or clinical and epidemiological studies. 


\section{Flavonoids, a decade of debate}

Over the past decade, many researchers have described the necessary requirements for establishing flavonoid bioactivity. The following appraisal details an extraction of a few important reviews conducted over the past decade and summarises the views offered by many experts in the field with regard to these requirements.

\section{$1999-2000$}

As suggested almost 10 years ago by Scalbert \& Williamson $^{(6)}$, when flavonoid research was just coming into the mainstream

Biologists should focus less on the parent compounds as they are ingested and more on the biological activities of the metabolites present in our tissues, and in particular on the conjugated analogues.

Today, 10 years later, many researchers are still investigating the biological activity of flavonoids in their parent forms, and we are no closer to understating the bioactivity, bioavailability, metabolism and pharmacokinetics of many of the flavonoid species. Similarly, Lapidot et $a l^{(12)}$ focused on chemical derivatives of anthocyanins rather than metabolites and suggested that $\mathrm{pH}$-dependent carbinol pseudo-base, quinoidal-base or the chalcones could be absorbed from the gut and present in the blood, and that these should be a focus of future research. However, information regarding the extent to which chemical derivatives may contribute to bioavailability or bioactivity of anthocyanins or other flavonoids is still unknown. In fact, despite advances beyond the analytical capacity of HPLC-diode array detection as a means of detection, these questions remain unanswered and under-investigated to this day.

The review by Scalbert \& Williamson ${ }^{(6)}$ as previously discussed also outlined the

need to better assess the role of the microflora in the bioavailability of polyphenols and to determine the proportions of the plasma phenolic metabolites absorbed by the small intestine or by the colon after transformation by the microflora.

With the exception of a limited few subclasses, very little is known about the complete metabolic pathway for most flavonoids in human subjects, primarily as a result of a lack of well-controlled labelling studies characterising recovery in serum, urine, faeces and expired air $\left(\mathrm{CO}_{2}\right)$.

\section{4}

In a review by Kroon et al. ${ }^{(13)}$, the researchers commented that

the past few years have seen very significant advances in our understanding of polyphenol metabolism. However, controversy remains concerning the nature and properties of flavonoid conjugates in vivo, and that uncertainty hampers progress toward understanding the real contribution of flavonoids as dietary protective agents against cancer, CVD and other diseases.
The authors further stated it is critical that all future studies attempting to use in vitro models to assess the effects of polyphenols in human subjects use physiologic conjugates at appropriate concentrations; ... and these

are key prerequisites to an understanding of the role of dietary polyphenols in human health.

Today, this plea has yet to be realised. The review further concluded that the greatest limitation in flavonoid research was the 'superficial' description of bioactivity based on studies using flavonoids in non-physiologically relevant forms or concentrations.

\section{5}

In a two-part supplement led by Manach et al. ${ }^{(7)}$, the authors explored the available studies on bioavailability and bioefficacy of flavonoids. The first supplement, by Manach et al., reviewed bioavailability studies and concluded that

the nature of the known metabolites is described when data are available. But complete data are still too limited for many of the flavonoids.

They also conferred that these data are essential for the ${ }^{(7)}$

design and interpretation of intervention studies investigating the health effects of polyphenols.

Today, progress in this area is slow and as stated throughout the above synopsis, more complex metabolic interventions involving labelled compounds are required.

In the second review of the two-part supplement by Williamson \& Manach $^{(4)}$, the authors stated that

compared with the effects of polyphenols in vitro, the effects in vivo, although significant, are more limited.

Furthermore, they stated that the reasons for this are $\mathrm{a}^{(4)}$

lack of validated in vivo biomarkers, especially in the area of carcinogenesis; a lack of long-term studies; and a lack of understanding or consideration of bioavailability in the in vitro studies.

Again, these issues are as relevant today as they were 5 years ago. The review further identified clear gaps in the literature between human intervention and mechanism of action, which resulted from too few studies demonstrating a dose-response relationship and previous studies being of too short a duration, which is essential for convincing evidence.

In 2005, the Proceedings of the ILSI North America Flavonoids Workshop (Washington, DC) was released ${ }^{(5)}$, which gave an overview of the present research on flavonoids with the goal of providing aid in establishing dietary recommendations and prioritising future research needed to establish the relationship between flavonoids and heart health. Within this document, the authors suggested that

the accurate determination of polyphenol intake is hindered by the lack of comprehensive food composition databases.

They also listed challenges for developing a food composition database for flavonoids, which included ${ }^{(5)}$ 
structural diversity of the compounds, the large number of dietary sources, the large variability in polyphenol content for a given source, the diversity of analytical methods, and in some cases, the lack of suitable analytical methods.

Although the United states Department of Agriculture and other databases have made significant progress in this area since this review was published, these databases are still a long way from being complete (i.e. including all flavonoid subclasses and common food sources).

Lastly, the review also focused on the lack of knowledge regarding the safety of flavonoid consumption in light of potential overconsumption in the case of supplementation or fortification. Although long-term consumption of relatively low-dose flavonoids from food sources is obviously safe within the context of our 'natural'/habitual diets, adverse effects of flavonoids from some foods have been observed and need to be a focus of future research ${ }^{(5)}$.

\section{8}

More recently, a review by Spencer et al. ${ }^{(9)}$ focused on biomarkers of polyphenol intake in nutrition research. In the present review, the authors focused on the necessity of accurate quantitative data regarding dietary intake in order to establish firm evidence for health claims. In addition, they stated that ${ }^{(9)}$

the usefulness of the current methods, which rely mainly on the assessment of polyphenol intake using food records and food composition tables, is limited as they fail to estimate total intake accurately.

Obviously, based on the above summary of reviews, this opinion is by no means novel, but one that has remained virtually unchanged over the past 10 years. And although progress has been made in this area, there is still a great deal more work to be done. Spencer et al. also highlighted the importance of establishing quality biomarkers of intake, dose response and pharmacokinetics, metabolic variability, effects of food matrix on bioavailability, contribution of colonic microflora to bioavailability and the importance of realising the metabolic fate of polyphenols. Again, these concepts have been preserved for many years.

\section{A decade of debate in summary}

Requirements for establishing flavonoid bioactivity are the following:

(1) establish differential effects of food processing and food matrix on bioavailability;

(2) develop more comprehensive food composition databases;

(3) develop validated, standardised and up-to-date methods of analysis;

(4) develop validated in vivo biomarkers of intake and activity;

(5) establish dose response and safe dosages;

(6) conduct more long-term dietary intervention trials (months to years);

(7) establish bioavailability and the impact of common metabolic pathways;
(8) establish all metabolic (including colonic) and chemical derivatives;

(9) develop in vitro models of bioactivity using physiologically appropriate conjugates (forms and concentrations).

\section{Present}

The aim of the present thesis was to outline where we have been, where we are today and where we need to be in the future. Although we have made significant advances in specific areas of polyphenol research, the question remains, why have we not been able to resolve the clear and persistent limitations within the field? Below, I present three possibilities, which are by no means a complete list of all probable explanations.

First, investigators are reluctant to pursue research into the fundamentals of flavonoid bioavailability using pure labelled compounds, over cheaper and more accessible work using crude flavonoid extracts and flavonoid containing foods. Much of this is likely a result of the difficulties in acquiring both funding and ethical permission for such projects. In order to resolve this issue, funding bodies need to focus on funding works, which aim to initially explore the fundamental aspects of polyphenol toxicology/pharmacology over more superficial studies only exploring the effect on biomarkers of disease. This drive to investigate end-stage health effects superficially has undoubtedly been driven by interest (academic, industrial, agricultural and consumer) in nutraceuticals and functional foods.

Second, there is an inherent drive to study the bioactivity of parent compounds in vitro, over studies using synthetically or biologically synthesised metabolites. This again is most likely the result of the inherent difficulties with synthesis and the associated time and costs.

Lastly, a persistent focus on research into the activities of flavonoids as direct radical scavengers (i.e. antioxidants) has undoubtedly delayed the progress of research in this field. Again, even though this direction of research has not been fruitful, the concept has been, and is still today, driven by consumer/commercial interest in antioxidants as nutraceuticals and functional foods. New focus into the actions of flavonoids on the expression of antioxidant enzymes through interactions with antioxidant response element genes may prove to be a more fruitful direction for future flavonoid research in this area. It should be noted that this is just one example of the potential mechanisms of flavonoid bioactivity, and many others have been implicated throughout the literature.

\section{Future}

The future of flavonoid research will undoubtedly depend on resolving the issues outlined above; and although the field has shown continuous progress for many years, progress will likely slow if these challenges are not met. If we follow the published advice, we should be imposing a more advanced model for flavonoid research, one which goes beyond our current and somewhat flawed approach. This model needs to include processing effects, dietary and bioavailable dose, absorption, metabolism and pharmacokinetics, physiologically appropriate in vitro models of bioactivity, and including a 
Model 1

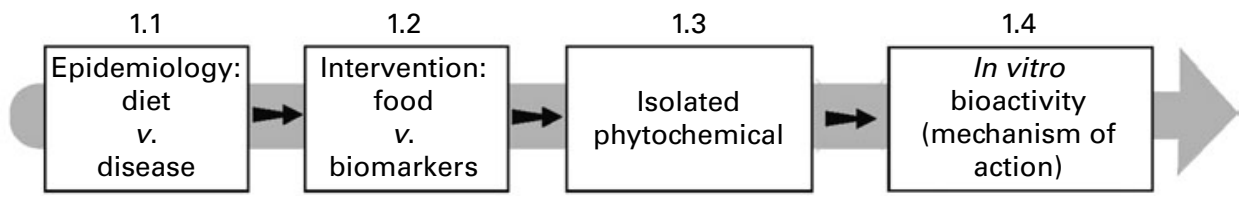

Fig. 1. Model 1.

focus on the structural relationships between the flavonoid subclasses. Lastly, the data derived from such a model should be used to inform future clinical studies using appropriate flavonoids, sources and disease end points.

The future of flavonoid research therefore requires a multi-disciplinary approach involving epidemiological, human intervention and cellular/molecular study. Within the current model (Fig. 1) as commonly utilised by most, a hypothesis is developed based on epidemiological associations between a disease outcome (such as CVD or cancer; 1.1) and the consumption of a food high in flavonoids. Then, intervention studies are conducted using these identified foods to examine the impact of the intervention on biomarkers of health/disease (e.g. cholesterol, blood pressure, oxidation; 1.2). Next, the flavonoids of interest are isolated from the food (1.3) and used to explore potential mechanisms of action in in vitro (or cell-based) models of bioactivity (1.4).

The key problems with model 1 are as follows: there are too many potential bioactive compounds in foods to associate any one with the observed outcome in epidemiological studies or dietary interventions using whole foods; isolated compounds from whole/fresh foods often do not represent the actual forms in processed foods, as commonly consumed in the diet; and the 'leap' from food-based phytochemical to in vitro/or cell-based mechanism of action (1.4) ignores many inherent biological complexities, including structural modification during absorption from the gastrointestinal tract, metabolism and concentration at the plasma cell/tissue interface. Consequently, the findings derived from exploiting the current 'commonly utilised' model have proven erroneous.
Therefore, a more advanced model for investigating the health effects of flavonoids must be explored, as proposed in Fig. 2 (model 2).

The proposed model (model 2) still utilises all the steps within model 1 , as these are useful in developing hypotheses and refining methods. However, model 2 does not stop at testing the compounds as they occur in foods (such as the parent/ precursor compounds in fruits and vegetables), but further establishes the structural modifications to these compounds occurring both during food processing (2.3.1) and human absorption and metabolism (2.3.2). In addition, the proposed model involves establishing the pharmacokinetics and metabolism of all circulating compounds post consumption (2.3.3), and tests their by-products of metabolism for biological activity (2.4).

The key benefits of the proposed approach (model 2) are that it accounts for structural modification to the bioactive compounds occurring during food processing; it establishes structural modification to the compound occurring during absorption and metabolism; it identifies physiologically relevant forms for synthesis; and it establishes the concentration of circulating metabolites, so physiologically relevant structures and concentrations can be applied to in vitrol (or cell culture) studies of bioactivity. In conclusion, the proposed model provides a more direct approach to the investigation of flavonoid bioactivity.

It is necessary to point out the many challenges with implementing the proposed research model: first, post consumption, flavonoids possess very complex conjugate profiles, making it difficult to isolate, identify and quantify any individual

\section{Model 2}

2.1

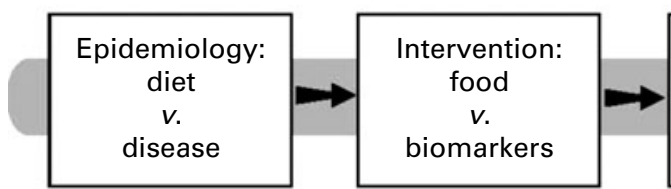

2.3

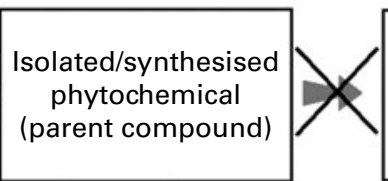

2.4

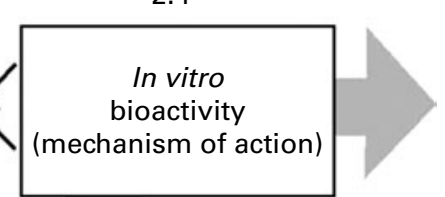

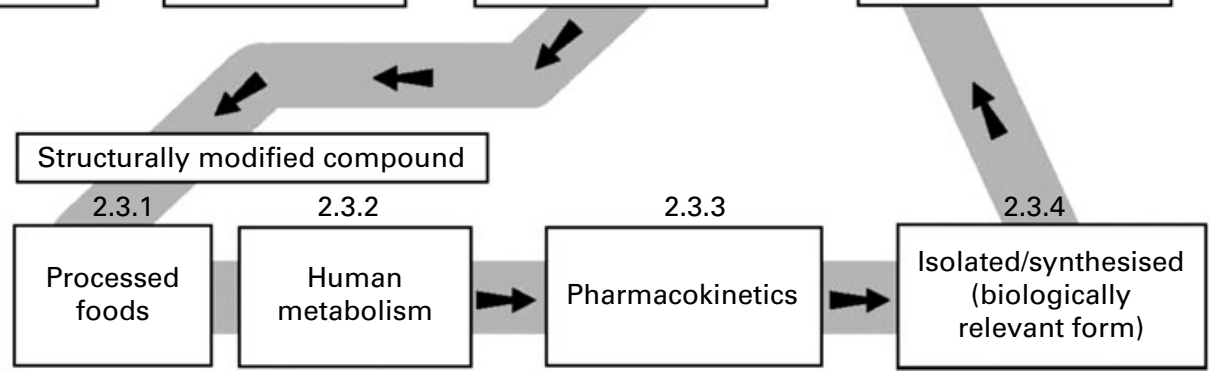

Fig. 2. Model 2. 
structure. Furthermore, based on the lack of available analytical standards, this will be a significant undertaking, one which requires an amplified synthetic or biosynthetic focus. Second, establishing bioavailability in most cases requires costly human studies using labelled compounds, which are inherently difficult to synthesise and expensive to make/purchase. Third, there is an endless variety of possible end points for the demonstration of biological effects, many of which are not validated or standardised.

\section{Implications}

If commercial/industrial and academic research councils fund programmes that attempt to address all stages of the proposed model, we will ultimately be able to conclusively characterise the structural forms of flavonoids as they occur in the body, so they may be applied in appropriate levels and forms to experimental models of biological activity. This will provide incite into the understanding of how flavonoids contribute to a healthy diet and how they may be utilised in the prevention or treatment of disease. This will also provide future commercial/economic benefits through aiding the establishment of 'new' functional ingredients or re-establishing old functional ingredients, and providing evidence for future health claims, thus helping to establish foods and their constituent phytochemicals as functional components for optimising human health.

\section{Acknowledgements}

The present review was supported in partnership with GlaxoSmithKline plc. (UK). The conditions laid down in the full Directions to Contributors are accepted, and the submission represents original work that has not been published previously and which is not currently being considered by another journal. The author reports no conflicts of interest. The consultancy support was provided by GlaxoSmithKline plc. (UK).

\section{References}

1. Mink PJ, Scrafford CG, Barraj LM, et al. (2007) Flavonoid intake and cardiovascular disease mortality: a prospective study in postmenopausal women. Am J Clin Nutr 85, 895-909.

2. Mursu J, Voutilainen S, Nurmi T, et al. (2008) Flavonoid intake and the risk of ischaemic stroke and CVD mortality in middleaged Finnish men: the Kuopio Ischaemic Heart Disease Risk Factor Study. Br J Nutr 100, 890-895.

3. Hooper L, Kroon PA, Rimm EB, et al. (2008) Flavonoids, flavonoid-rich foods, and cardiovascular risk: a meta-analysis of randomized controlled trials. Am J Clin Nutr 88, 38-50.

4. Williamson G \& Manach C (2005) Bioavailability and bioefficacy of polyphenols in humans. II. Review of 93 intervention studies. Am J Clin Nutr 81, 243S-255S.

5. Erdman JW Jr, Balentine D, Arab L, et al. (2007) Flavonoids and Heart Health: proceedings of the ILSI North America Flavonoids Workshop, May 31-June 1, 2005, Washington, DC. $J$ Nutr 137, 718S-737S

6. Scalbert A \& Williamson G (2000) Dietary intake and bioavailability of polyphenols. J Nutr 130, 2073S-2085S.

7. Manach C, Williamson G, Morand C, et al. (2005) Bioavailability and bioefficacy of polyphenols in humans. I. Review of 97 bioavailability studies. Am J Clin Nutr 81, 230S-242S.

8. Kuhnau J (1976) The flavonoids: a class of semi-essential food components: their role in human nutrition. Rev Nutr Diet 24, $117-191$

9. Spencer JP, Abd El Mohsen MM, Minihane AM, et al. (2008) Biomarkers of the intake of dietary polyphenols: strengths, limitations and application in nutrition research. Br J Nutr 99, $12-22$.

10. U.S. Department of Agriculture, Agricultural Research Service (2009) USDA National Nutrient Database for Standard Reference, Release 22. Nutrient Data Laboratory Home Page. http://www.ars.usda.gov/ba/bhnrc/ndl

11. EuroFir (2009) European Food Information Resource Network Food Composition Databank. http://www.eurofir.net/index. asp? $\mathrm{id}=1$

12. Lapidot T, Harel S, Akiri B, et al. (1999) pH-dependent forms of red wine anthocyanins as antioxidants. J Agric Food Chem 47, 67-70.

13. Kroon PA, Clifford MN, Crozier A, et al. (2004) How should we assess the effects of exposure to dietary polyphenols in vitro? Am J Clin Nutr 80, 15-21. 